\title{
ANTIOXIDANTS, RADICAL-SCAVENGING AND PROTEIN CARBONYLATION INHIBITION CAPACITY OF SIX MONOCULTIVAR VIRGIN OLIVE OILS IN ISTRIA (CROATIA)
}

\author{
O. KoprivnjaK ${ }^{\mathrm{a}}$, A. KrišKo ${ }^{\mathrm{b}}$, S. Valićc, D. Carićc ${ }^{\mathrm{c}}$, M. Krapac ${ }^{\mathrm{d}}$ and D. Poljuha ${ }^{\mathrm{d}, e *}$

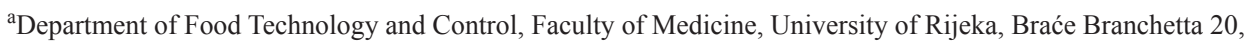 \\ 51000 Rijeka. Croatia \\ ${ }^{\mathrm{b}}$ MedILS-Mediterranean Institute for Life Sciences, Meštrovićevo šetalište 45, 21000 Split. Croatia \\ ${ }^{\mathrm{c}}$ Ruđer Bošković Institute, Bijenička cesta 54, 10000 Zagreb. Croatia \\ ${ }^{\mathrm{d} I n s t i t u t e}$ of Agriculture and Tourism, Karla Huguesa 8, 52440 Poreč. Croatia \\ ${ }^{\mathrm{e}}$ Materials Research Centre METRIS, Istrian Development Agency, Zagrebačka 30, 52100 Pula, Croatia
}

(Received: 4 December 2014; accepted: 2 January 2015)

Six monocultivar virgin olive oils (VOOs) produced from five autochthonous (Buža, Buža Puntoža, Istarska Bjelica, Porečka Rosulja, and Rosinjola) and one referent cultivar (Leccino), were investigated. The mass fractions of orthodiphenols (ORT) and tocopherols (TOC) were analysed by VIS spectroscopy and HPLC. The radical-scavenging capacity was evaluated by electron spin resonance spectroscopy (ESR) using galvinoxyl free radical and the DPPH test. Results revealed a high level of total TOC in VOOs of Buža Puntoža (243 mg kg-1) and Porečka Rosulja (325 $\mathrm{mg} \mathrm{kg}^{-1}$ ). VOOs contained in $100 \mathrm{~g}$ from $79 \%$ (Istarska Bjelica) to 261\% (Porečka Rosulja) of recommended daily allowance of $\alpha$-tocopherol. The mass fraction of ORT in Istarska Bjelica and Rosinjola was $>250 \mathrm{mg} \mathrm{kg}^{-1}$ (on average 30-40\% higher in comparison to other studied cultivars). Istarska Bjelica and Porečka Rosulja showed the highest antioxidant capacity in both methods of measurement. The highest capacity to inhibit protein carbonylation (PC) in response to oxidative stress (54-56\%) was displayed by Buža Puntoža, Leccino, and Buža. High level of positive linear correlation between ORT mass fraction and radical-scavenging capacity measured by DPPH test $(\mathrm{r}=0.768)$, as well as strong negative correlation between PC inhibition and mass fraction of ORT $(\mathrm{r}=-0.697)$, were observed.

Keywords: virgin olive oil, ortho-diphenols, tocopherols, antioxidant capacity, protein carbonylation.

Tocopherols and hydrophilic phenols are the main natural antioxidants in virgin olive oils (VOO) (Boskou, 2009). Their natural content essentially determines the nutritional value of the individual $\mathrm{VOO}$, since it is a food product that is not allowed to be enriched by adding of any nutrient (EEC, 1991). Mass ratio of total tocopherols in VOO generally ranges from 100 to $300 \mathrm{mg} \mathrm{kg}^{-1}$ (APARicio \& LunA, 2002; Boskou et al., 2006; TurA et al., 2007). Within this group of lipophilic substances owning vitamin and antioxidant activity, the predominant form is $\alpha$-tocopherol, characterized by the highest biological value (VALK \& HorNSTRA, 2000). Mass ratio of total hydrophilic phenols in VOO occurs in the range of 50 to 1000 $\mathrm{mg} \mathrm{kg}^{-1}$ (Boskou, 2009). In addition to the proven direct antioxidant effect and contribution to the oxidative stability of oil (CARRASCO-PANCORBO et al., 2005), these substances are associated with certain beneficial health effects (BENDINI et al., 2007).

\footnotetext{
* To whom correspondence should be addressed.

Phone: +38-552-408300; fax: +38-552-431659; e-mail: danijela@iptpo.hr
} 
The above-mentioned ranges of hydrophilic phenols and tocopherols mass ratio indicate considerable variability of VOO composition. This composition could be affected not only by maturity and integrity of fruit, climatic and meteorological conditions of olive tree cultivation, and applied oil extraction technology, but also to a great extent by genetic factors (ApAricio \& LunA, 2002; Tura et al., 2007; ŽAnetić et al., 2013). From the nutritional aspect, it could be useful to select olive cultivars with genetic potential for high level of natural antioxidants in oil. Autochthonous cultivars from smaller breeding areas, being less widespread and still scarcely studied, represent an interesting resource for exploring such potential. Therefore, five autochthonous cultivars in Istria (Buža, Buža Puntoža, Istarska Bjelica, Porečka Rosulja, and Rosinjola) and one referent cultivar (Leccino) have been taken in consideration in this study. All six cultivars were subjected to the same growing and processing conditions, thus focusing on genetic specificity as the main source of differences.

Beside antioxidants content, antioxidant capacity of oil samples measured by electron spin resonance spectroscopy (ESR) and by VIS spectroscopy using DPPH free radical was determined in reaction media of different polarity that can influence the activity of lipophilic and hydrophilic antioxidants (HuANG et al., 2005). Regarding oxidative stress in organism, one of the most common indicators is the oxidative carbonylation of proteins (CASTEGNA et al., 2003). Therefore, the capacity of six monocultivar VOOs to reduce protein carbonylation in response to oxidative stress, based on quantitative immunodetection of carbonyl groups introduced into proteins by metal catalysed oxidation, was measured as well.

\section{Materials and methods}

\subsection{Olive fruit and preparation of virgin olive oil samples}

Fruit were handpicked on the Istrian west coast area in October and November 2011, at the same maturity index $(\mathrm{MI}=2.5-3.0)$, except Istarska Bjelica $(\mathrm{MI}=1.0)$. MI (scale ranging from 0 to 7) was determined according to GuTIÉRREZ and co-workers (1999). Fruit samples were processed within $24 \mathrm{~h}$ after harvesting by using hammer crusher, vertical mixers thermostated at $26 \pm 0.5^{\circ} \mathrm{C}$, and centrifuge (MC2 Ingenieria y Sistemas, Seville, Spain). Extraction yields per fruit's fresh weights were as follows: 5.7\% (Buža), 6.8\% (Buža Puntoža), 17.3\% (Istarska Bjelica), 9.8\% (Leccino), 8.9\% (Porečka Rosulja), and 8.1\% (Rosinjola). The extracted oil was filtered through the filter paper and stored at room temperature in sealed dark bottles until analyses. Two repetitions of oil extraction procedure per single cultivar were done.

\subsection{Determination of tocopherols}

Tocopherols were analysed by HPLC (Varian ProStar HPLC, fluorescence detector) according to the standard method (ISO, 1997). The individual tocopherols were separated on a Restek Pinnacle II silica column, $15 \mathrm{~cm} \times 4.6 \mathrm{~mm}$ i.d. $(5 \mathrm{~mm})$. Standards of $\alpha$-, $\gamma$-, and $\delta$-tocopherol were used for the setup of calibration curves ( 5 to $750 \mathrm{mg} \mathrm{kg}^{-1}$ of tocopherols in oil). Each sample was analysed in duplicate.

\subsection{Determination of ortho-diphenols}

The procedure proposed by International Olive Council (IOC, 2007), which includes solid phase extraction and measurement of absorbance at $370 \mathrm{~nm}$, was applied. Each sample was analysed in duplicate. The results were expressed as pyrocatechol equivalents. 


\subsection{ESR measurements of radical-scavenging capacity}

Measurements were performed at $22{ }^{\circ} \mathrm{C}$ using a Varian E-109 spectrometer with a Bruker ER $041 \mathrm{XG}$ microwave bridge. The spectroscopic parameters were: frequency $9.27 \mathrm{GHz}$, field sweep $10 \mathrm{mT}$, microwave power $4.9 \mathrm{~mW}$, and modulation amplitude $0.11 \mathrm{mT}$. Samples were prepared according to PAPADIMITRIOU and co-workers (2006). The freshly prepared $0.3 \mathrm{mM}$ galvinoxyl free radical (GFR) solution in isooctane was added to the VOO in order to obtain a $4 \%(\mathrm{w} / \mathrm{v})$ oil solution, mixed in the flask for $10 \mathrm{~s}$ and then put into the standard ESR tube. The signal intensities of GFR were calculated by the double integration of ESR spectra, using the EPRWare Scientific Software Service programme and expressed in arbitrary units. The signal intensity of the pure $0.3 \mathrm{mM}$ GFR solution was taken as the signal intensity of the sample $\left(A_{0}\right)$ for the reaction time zero $(\mathrm{t}=0 \mathrm{~min})$. The loss of the signal intensity (l) was calculated as: $1=\left[\left(\mathrm{A}_{0}-\mathrm{A}\right) / \mathrm{A}_{0}\right] \times 100 \%$, where $\mathrm{A}$ is the signal intensity of GFR in oil solution measured $90 \mathrm{~min}$ after the oil and GFR solution contact.

\subsection{Measurements of radical-scavenging capacity using VIS spectroscopy}

The reaction mixture of oil and DPPH·(2,2'-diphenyl-1-picrylhydrazyl) solution was prepared according to KALANTZAKIS and co-workers (2006). One $\mathrm{ml}$ of oil solution $(10 \%$, w/v in ethyl acetate) was added to $4 \mathrm{ml}$ of a freshly prepared DPPH solution $(0.1 \mathrm{mM}$ in ethyl acetate) in $20 \mathrm{ml}$ test tube, which was immediately tapped and vigorously mixed for $10 \mathrm{~s}$. Absorbance was measured at $515 \mathrm{~nm}$, using a HACH spectrophotometer DR/400, (Colorado, USA) at 90 min after the oil and free radical solution contact. The absorbance for $t=0\left(A_{0}\right)$ was calculated as: $A_{0}=A_{B}+A_{S}$, where $A_{B}$ is the absorbance of blank solution (DPPH $0.1 \mathrm{mM}$ ) and $A_{\mathrm{S}}$ is absorbance of oil solution $(10 \%, \mathrm{w} / \mathrm{v})$. The loss of DPPH $\cdot$ absorbance $(l)$ was calculated as described for the loss of signal intensity in ESR measurements. Each sample was analysed in duplicate.

\subsection{Measurements of protein carbonylation}

Protein carbonylation (PC) was determined by OxyELISA Oxidized Protein Quantitation kit (Millipore), following the kit instructions. The model protein ( $\alpha$-synuclein) diluted to $10 \mu \mathrm{g} \mathrm{m} l^{-1}$ was loaded into micro titer plate wells and incubated overnight at $4{ }^{\circ} \mathrm{C}$. Then oil samples $(10 \mu \mathrm{l})$ were added, followed by incubation with oxidation solution $(25 \mathrm{mM}$ HEPES, $25 \mathrm{mM}$ sodium ascorbate, $0.1 \mathrm{mM} \mathrm{FeCl}_{3}$ ) for $5 \mathrm{~min}$ at room temperature and detection of derivatised dinitrophenol (DNP)-carbonyl by a rabbit DNP specific monoclonal antibody and secondary goat anti rabbit antibody conjugated to horseradish peroxidase. After incubation with enzyme substrate 3,3',5,5'-tetramethylbenzidine, a coloured product was quantified using a microplate reader at $450 \mathrm{~nm}$. The degree of PC inhibition achieved in presence of oil samples (I) is expressed as a percentage relative to the control (trial without oil sample) and was calculated as: $I=100-A_{S} \times 100 / A_{C}$, where $A_{S}$ is the absorbance in presence of oil sample, while $A_{C}$ is the absorbance of control trial. Each sample was analysed ten times.

\subsection{Statistics / Data analysis}

Differences among VOO samples were tested by the one-way analysis of variance at $5 \%$ significance level. The homogeneity of variance was tested by the Brown-Forsythe test. The mean values were compared by the Tukey's honest significant difference test $(\mathrm{P} \leq 0.05)$. 
Statistical analyses were performed using the software package Statistica version 10 (StatSoft, Tulsa, OK, USA).

\section{Results and discussion}

Mass fractions of tocopherols in oils of six olive cultivars are presented in Fig. 1. In comparison to literature data, Porečka Rosulja, Leccino, and Buža Puntoža could be considered as cultivars characterised by high levels of total tocopherols (243-325 mg kg-1). According to TURA and co-workers (2007), Leccino is one of the richest among several Italian cultivars regarding mass fraction of total tocopherols. Similar was found by KOPRIVNJAK and co-workers (2012) for Leccino cultivated in 2010 and 2011 in Istria, in comparison with Istarska Bjelica and Buža. However, no literature data related to Porečka Rosulja and Buža Puntoža are published so far and results obtained in this study suggest their great potential in production of VOOs rich in tocopherols. The ratio of $\alpha$-tocopherol in total tocopherols in oils of six cultivars ranged from $95.1 \%$ (Buža Puntoža) to $99.5 \%$ (Buža) that is quite high when compared to literature data (Boscou, 2009). Oil samples contained from 79\% (Istarska Bjelica) to $261 \%$ (Porečka Rosulja) of recommended daily allowance of $\alpha$-tocopherol (12 $\mathrm{mg}$ ) in $100 \mathrm{~g}$. According to criteria accepted by European Union authorities (EC, 2006), all of them can be considered rich sources of vitamin E (food containing at least $30 \%$ of recommended daily allowance in $100 \mathrm{~g}$ ).

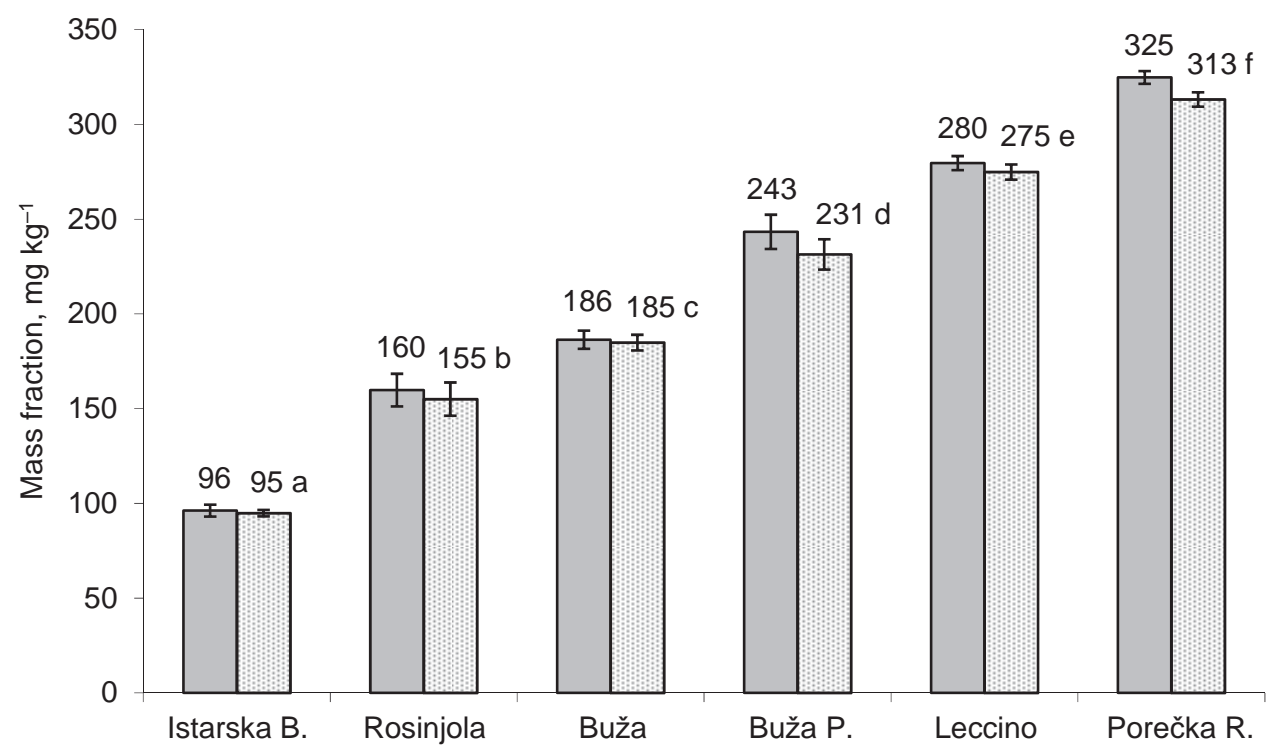

Fig. 1. Mass fraction of tocopherols in VOO samples. Results are mean values of four measurements ( 2 independent repetitions of oil extraction procedure $\times$ duplicate analyses) \pm S.D.; the mean values labelled with different letters are significantly different (Tukey's test, $\mathrm{P}<0.05$ ). $\square$ : total tocopherols; $\$$ : $\alpha$-tocopherol

As regards ortho-diphenols mass fraction (Fig. 2), it can be seen that Istarska Bjelica and Rosinjola had on average from 30 to $40 \%$ higher values regarding other studied cultivars. Literature data related to these cultivars report mainly values of total hydrophilic phenols in 
oil (Poljuha et al., 2008; Koprivnjak et al., 2012), and confirm that they have the highest potential in production of VOOs rich in these compounds. According to European Union authorities, hydrophilic phenols in VOO and particularly ortho-diphenols, contribute to the protection of blood lipids from oxidative stress when provided by daily consumption of $20 \mathrm{~g}$ of VOO containing at least $5 \mathrm{mg}$ ( $\geq 250 \mathrm{mg} \mathrm{kg}^{-1}$ ) of these compounds (EC, 2012). Considering ortho-diphenols, Istarska Bjelica and Rosinjola are only two out of six studied cultivars that complete the criteria for such health claim. However, it must be taken into account that mentioned health claim limit is related to mass fraction of total hydrophilic phenols.

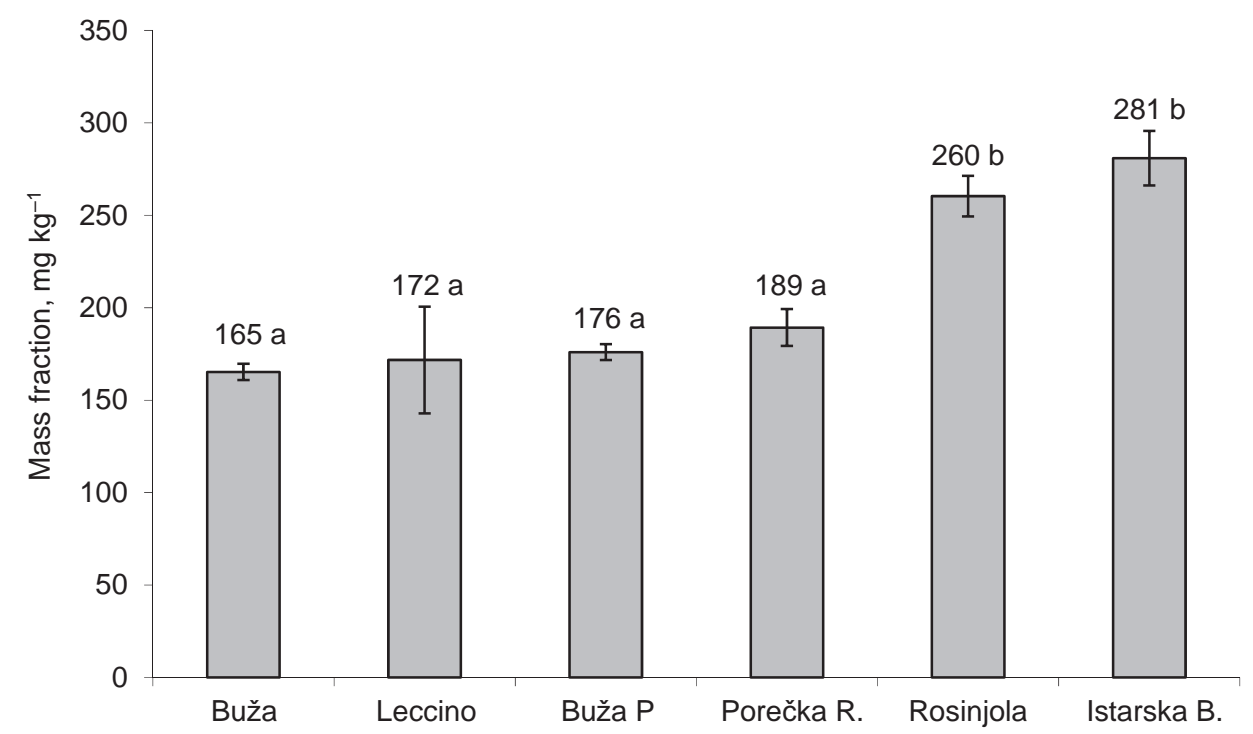

Fig. 2. Mass fraction of ortho-diphenols in VOO samples. Results are mean values of four measurements ( 2 independent repetitions of oil extraction procedure $\times$ duplicate analyses $) \pm$ S.D.; the mean values labelled with different letters are significantly different (Tukey's test, $\mathrm{P}<0.05$ )

Istarska Bjelica and Porečka Rosulja showed the highest radical-scavenging capacity in both methods of measurement (Table 1). Istarska Bjelica (the richest in ortho-diphenols and the poorest in tocopherols) and Porečka Rosulja (relatively low mass fraction of orthodiphenols and the highest of tocopherols) were quite different as regards content of two types of antioxidants. Mass fraction of tocopherols was very weakly correlated to radicalscavenging capacity in both non-polar (isooctane, EPR) and polar solvent surrounding (ethyl acetate, VIS). Ortho-diphenols showed strong positive correlation with radical-scavenging capacity in polar solvent and this suggests their strongest antioxidant activity in comparison to tocopherols in experimental conditions applied in this study. In fact, BALDIOLI and coworkers (1996) have shown that oleuropein and hydroxytyrosol are more effective than vitamin E regarding hydrogen-donating activity. 
Table 1. Radical-scavenging capacity and inhibition of protein carbonylation*

\begin{tabular}{lccc}
\hline Cultivars & \multicolumn{2}{c}{ Radical signal intensity loss $(\%)$} & $\begin{array}{c}\text { Inhibition of protein } \\
\text { carbonylation } \\
(\%)\end{array}$ \\
\cline { 2 - 3 } & $\begin{array}{c}\text { DPPH } \\
(\text { VIS })\end{array}$ & $\begin{array}{c}\text { Galvinoxyl } \\
(\mathrm{EPR})\end{array}$ & $54 \pm 4 \mathrm{a}$ \\
Buža & $45 \pm 2 \mathrm{a}$ & $60 \pm 1 \mathrm{a}$ & $56 \pm 3 \mathrm{a}$ \\
Buža Puntoža & $50 \pm 2 \mathrm{ad}$ & $65 \pm 1 \mathrm{~d}$ & $46 \pm 2 \mathrm{~b}$ \\
Istarska Bjelica & $74 \pm 4 \mathrm{~b}$ & $78 \pm 2 \mathrm{~b}$ & $55 \pm 3 \mathrm{a}$ \\
Leccino & $56 \pm 5 \mathrm{~cd}$ & $72 \pm 4 \mathrm{c}$ & $42 \pm 3 \mathrm{c}$ \\
Porečka Rosulja & $65 \pm 1 \mathrm{e}$ & $80 \pm 2 \mathrm{~b}$ & $35 \pm 6 \mathrm{~d}$ \\
Rosinjola & $60 \pm 1 \mathrm{c}$ & $75 \pm 2 \mathrm{bc}$ & 0.234 \\
$r$ tocopherols** & -0.270 & 0.064 & -0.697 \\
$r$ ortho-diphenols** & 0.768 & 0.187 & \\
\hline
\end{tabular}

*The results of VIS and EPR are expressed as mean of four values \pm SD (two repetitions of oil extraction procedure $\times$ two repetitions of analytical procedure), while the results of protein carbonylation as mean of twenty values $\pm \mathrm{SD}$ (two repetitions of oil extraction procedure $\times$ ten repetitions of analytical procedure). The means within each column marked with different letters are significantly different (Tukey's test, $\mathrm{P}<0.05$ ).

**Linear correlation coefficients between radical-scavenging capacity or protein carbonylation reduction and tocopherols or ortho-diphenols mass fraction.

The capacity of the VOOs to inhibit the occurrence of protein carbonylation was studied in vitro by using $\alpha$-synuclein, a protein expected to be highly susceptible to PC due to its disordered structure. Under such conditions, the highest inhibition rates of $\alpha$-synuclein carbonylation was displayed in the presence of Buža Puntoža, Leccino, and Buža (all above $50 \%$ compared to the control), without statistically significant differences among them. Linear correlation coefficient between content of tocopherols and PC inhibition capacity showed positive but a fairly low value. Surprisingly, mass fraction of ortho-diphenols in oil samples was negatively correlated to PC inhibition capacity at quite high level. Considering this, the antagonistic effect between these two natural antioxidants could not be excluded. Moreover, taking into account the complex composition of VOOs, different relationships among potentially active components, both synergism and antagonism, could be expected.

\section{Conclusions}

The results suggest that, among cultivars considered in this study, Istarska Bjelica, Porečka Rosulja, and Rosinjola have the highest potential in production of VOOs rich in antioxidants and with strong antioxidant capacity, although with less expressed inhibition of protein carbonylation. From this point of view, these three cultivars could be recommended to olive growers for propagation and cultivation, in order to make the product competitive in the global market.

This work was partly supported by the Istrian Development Agency (IDA), Istrian County and by the University of Rijeka under the project number 13.06.1.4.50. 


\section{References}

Aparicio, R. \& LunA, G. (2002): Characterisation of monovarietal virgin olive oils. Eur. J. Lipid Sci. Tech., 104, 614-627.

Baldioli, M., Servili, M., Perretti, G. \& Montedoro, G. (1996): Antioxidant activity of tocopherols and phenolic compounds in virgin olive oil. J. Am. Oil Chem. Soc., 73, 1589-1593.

Bendini, A., Cerretani, L., Carrasco-Pancorbo, A., Gómez-Caravaca, A.M., Segura-Carretero, A., FernándezGutiérrez, A. \& Lercker, G. (2007): Phenolic molecules in virgin olive oils: A survey of their sensory properties, health effects, antioxidant activity and analytical methods. An overview of the last decade. Molecules, 12, 1679-1719.

Boskou, D., Tsimidou, M. \& Blekas, D. (2006): Olive oil, chemistry and technology. AOCS Press, Champaign, USA, p. 44.

Boskou, D. (2009): Phenolic compounds in olives and olive oil. -in: Boskou, D. (Ed.) Olive oil minor constituents and health. CRC Press, Boca Raton, USA, pp. 11-44.

Carrasco-Pancorbo, A., Cerretani, L., Bendini, A., Segura-Carretero, A., Del Carlo, M., Gallina-Toschi, T., Lercker, G., Compagnone, D. \& Fernández-Gutiérez, A. (2005): Evaluation of the antioxidant capacity of individual phenolic compounds in virgin olive oil. J. Agr. Food Chem., 53, 8918-8925.

Castegna, A., Drake, J., Pocemich, C. \& Butterfield, D.A. (2003): Protein carbonyl levels - An assessment of protein oxidation. -in: Hensley, K. \& Floyd, R.A. (Eds) Methods in biological oxidative stress: Methods in pharmacology and toxicology. Humana Press Inc., Totowa, NJ, pp. 161-168.

EC (2006): Regulation no. 1924/2006, O. J. L 404, p1 of 30 December 2006.

EC (2012): Regulation no. 432/2012, O. J. L 136, p1 of 16 May 2012.

EEC (1991): Regulation no. 2568/1991, O. J. L 248, p1 of 11 July 1991.

Gutiérrez, F., JímenEz, B., Ruíz, A. \& Albi, M.A. (1999): Effect of olive ripeness on the oxidative stability of virgin olive oil extracted from the varieties Picual and Hojiblanca and on the different components involved. J. Agr. Food Chem., 47, 121-127.

Huang, D., Ou, B. \& Prior, R.L. (2005): The chemistry behind antioxidant capacity assays. J. Agr. Food Chem., 53, 1841-1856.

IOC (2007). Colorimetric method for the determination of o-diphenolic compound in olive oils. International Olive Council. Available at: http://www.internationaloliveoil.org/estaticos/view/224-testing-methods (last accessed: 4 December 2014)

ISO (1997): Animal and vegetable fats and oils - determination of tocopherols and tocotrienols contents - method using high-performance liquid chromatography. ISO International Standard no. 9936, International Organization for Standardization, Geneva, Switzerland.

Kalantzakis, G., Blekas, G., Pegkildou, K. \& Boskou, D. (2006): Stability and radical-scavenging activity of heated olive oil and other vegetable oils. Eur. J. Lipid. Sci. Tech., 108, 329-335.

Koprivnjak, O., Vrhovnik, I., Hladnik, T., Prgomet, Ž., Hlevnjak, B. \& Majetić Germek, V. (2012): Characteristics of nutritive value of virgin olive oils from Buža, Istarska Bjelica, Leccino and Rosulja cultivars. Croat. J. Food Technol. Biotechnol., Nutr., 7, 172-178.

Papadimitriou, V., Sotiroudis, T.G., Xenakis, A., Sofikiti, N., Stavyiannoudaki, V. \& Chaniotakis, N.A. (2006): Oxidative stability and radical scavenging activity of extra virgin olive oils: An electron paramagnetic resonance spectroscopy study. Anal. Chim. Acta, 573, 453-458.

Poljuha, D., Sladonja, B., Brkić Bubola, K., Radulović, M., Brščić, K., Š́tić, E., Krapac, M. \& Milotić, A. (2008): A multidisciplinary approach to the characterisation of autochthonous Istrian olive (Olea europaea L.) varieties. Food Technol. Biotech., 46, 347-354.

Tura, D., Gigliotti, C., Pedò, S., Failla, O., Bass, D. \& Serraiocco, A. (2007): Influence of cultivar and site of cultivation on levels of lipophilic and hydrophilic antioxidants in virgin olive oils (Olea europaea L.) and correlations with oxidative stability. Sci. Hortic., 112, 108-119.

VAlk, E.E. \& Hornstra, G. (2000): Relationship between vitamin E requirement and polyunsaturated fatty acid intake in man: a review. Int. J. Vitam. Nutr. Res., 70, 31-42.

Žanetić, M., Cerretani, L., Š́kevin, D., Politeo, O., Vitanović, E., Jukić Š́pika, M., Perica, S. \& Ožić, M. (2013): Influence of polyphenolic compounds on the oxidative stability of virgin olive oils from selected autochthonous varieties. J. Food Agric. Environ., 11, 126-131. 